\title{
Diffusion Tensor Imaging in Mild Traumatic Brain Injuries - Acute State and Short-Term Recovery
}

\section{Corresponding author:}

Armin Fuchs

Center for Complex Systems \& Brain Sciences

Florida Atlantic University

777 Glades Road

Boca Raton, FL 33431

USA

Phone: +1-561-297-0125

Fax: +1-561-297-3634

Email: afuchs@fau.edu

\section{Authors:}

Armin Fuchs, Ph.D. ${ }^{1,2} \quad$ afuchs@fau.edu

Angelica Hotiu, Ph.D. ${ }^{2} \quad$ ahotiu@fau.edu

Kelly J. Jantzen, Ph.D. ${ }^{3} \quad$ kelly.jantzen@wwu.edu

Fred L. Steinberg, M.D. ${ }^{1,4} \quad$ fsteinberg@universitymri.com

J.A. Scott Kelso, Ph.D. ${ }^{1,5} \quad$ kelso@ccs.fau.edu

\section{Affiliations:}

${ }^{1}$ Center for Complex Systems \& Brain Sciences

Florida Atlantic University

777 Glades Road

Boca Raton, FL 33431

USA

${ }^{4}$ University MRI \& Diagnostic Imaging Centers 3438 FAU Blvd.

Boca Raton, FL 33431

USA

${ }^{2}$ Department of Physics

${ }^{5}$ Intelligent Systems Research Centre

Florida Atlantic University

University of Ulster

777 Glades Road

Boca Raton, FL 33431

Derry BT48 7JL

United Kingdom

USA

${ }^{3}$ Department of Psychology

Western Washington University

516 High Street

Bellingham, WA 98225 USA 


\section{Abstract}

Mild traumatic brain injuries (MTBI), in most cases, cannot be detected using imaging modalities like CT or MRI. However, diffusion tensor imaging (DTI) reveals subtle changes in white matter integrity as a result of head trauma and plays an important role in refining diagnosis and management of MTBI. We use DTI to detect the microstructural changes in collegial football players induced by axonal injuries and to monitor their evolution during the recovery process. Three players suffered a MTBI during play or practice and underwent scanning within $24 \mathrm{~h}$ with follow-ups after one and two weeks. Scalar diffusion indices were derived from diffusion tensors and analyzed using tract-based spatial statistics (TBSS) and voxel-wise t-tests to detect brain regions showing significant group differences between the injured subjects and controls. Both analyses revealed overlapping regions in the corticospinal tract with significant increase in fractional anisotropy and decreases in transverse and mean diffusivity within 24 hours. In voxel-wise t-tests strong indications for recovery were found spatially and temporally. For mean and transverse diffusivity, regions showing significant differences shrunk between the first and the follow-up scans. Although the sample size is small, these findings are remarkably consistent across all subjects and scans.

\section{Keywords:}

Diffusion tensor imaging (DTI), mild traumatic brain injuries (MTBI), fractional anisotropy (FA), diffusion indices

\section{Introduction}

Mild traumatic brain injuries (MTBI), or concussions, represent one of the most common types of head injury, which affect about 128 per 100,000 people in the US annually (Pearce, 2008; Ropper, Gorson, 2007). Despite the classification as mild, these injuries can develop significant post-concussive somatic (e.g. headache, dizziness) and affective (e.g. depression, irritability) symptoms, cognitive deficits (e.g. poor memory, difficulty concentrating) and motor dysfunctions, which may last more than a year in approximately $15-25 \%$ of the cases (Alves et al., 1993; Bazarian et al., 2005; Bazarian et al., 2007). Traumatic brain injuries constitute a significant cause of disability, thus it is essential to understand their pathophysiology in order to perform suitable diagnostic evaluations, intervene as early as possible and improve clinical treatment.
Among the traumatic brain injuries, those that occur as sports injuries play an important role due to the appreciable risk of sequelae, including persistent disabilities. In general, concussions are one of the most common and least serious traumatic brain injuries caused by a sudden blow to the head or body resulting in accelerating or decelerating forces without a direct impact on the brain. However, as a result of the biomechanical forces the brain shakes inside the skull, which may lead to unconsciousness. The shearing forces can disrupt cellular processes in the brain for days or weeks, produce diffuse axonal injuries and lead to a whole cascade of potentially harmful biochemical processes (Xiong et al., 2013).

Although concussions are transitory in most cases, they may have significant impact for neurological function. Thus, concussions may cause brain contusion, intracranial hemorrhage and axonal injury. Diffuse axonal injury is one of the most 
common pathologies in all types of traumatic brain injury (mild, moderate and severe) (Adams et al., 1989; Povlishock et al., 1983). Shearing forces acting on the brain during rapid acceleration or deceleration of the head can

cause severe axonal injury that is associated with unconsciousness and poor outcome. The axons' sensitivity to brain injury is due both to their viscoelastic properties and their highly organized structures in white matter tracts (Smith, Meany, 2000). Although axons are supple under normal conditions, shear forces produce rapid stretches or distortions that damage the axonal

cytoskeleton or disrupt the axons and small blood vessels. Axonal damage is related to the direction and magnitude of the shearing forces (Gennarelli, 1986). Whereas smaller forces lead to a potential recovery, greater forces can yield permanent loss of axonal functions.

Unfortunately, the microstructural changes that occur at different phases of the diffuse axonal injury process are not detectable using CT or conventional MRI. A more recent noninvasive imaging technique, diffusion tensor imaging (DTI), has a higher sensitivity for detection of axonal changes compared to MRI or CT scans. Initial DTI studies have shown signal abnormalities in subjects who suffered mild traumatic brain injuries but most of these studies examined the patients at different time points post-injury, thus comparison of the results is difficult. Moreover, there is a lack of longitudinal studies to monitor the evolution of diffusion indices during recovery and to describe possible changes in white matter integrity over time. The primary objective of this study was to demonstrate that changes in white mater integrity as a result of head trauma may be detected within 24 hours post injury and to compare these findings with follow-up scans at one and two weeks after the injury to monitor possible changes during the recovery process. To accomplish this objective we compared the scans from a control group to the sequence of three scans taken from injured subjects to detect potentially affected brain areas and their changes in time. In addition, we used a second control group of healthy subjects to show that our findings are due to the injury and not other factors, e.g. when the scans took place, which was spread over more than a year.

\section{Materials and Methods}

\subsection{Subjects}

The datasets used here were recorded within a larger study of MTBI in college football players that included functional and structural MRI as well as behavioral measures (Jantzen et al., 2000). The study was approved by Florida Atlantic University's Institutional Review Board and informed consent was obtained from all participants. The DTI data were acquired from 11 male intercollegiate football players of age 19-23 years (median age 20 years). Three players had suffered a mild traumatic brain injury during practice or play and underwent the scanning procedure within 24 hours after the injury with follow-ups after one and two weeks.

All DTI data analyses were performed on three groups of subjects: the concussed group, consisting of three injured players, and two control groups formed by randomly selecting five and three subjects from the pool of players that did not have a concussion. Throughout this article we shall refer to the concussed group as $\mathrm{CON}$ and to the control groups as CGA and CGB, respectively.

\subsection{DTI Acquisition}

DTI scanning was performed on a $1.5 \mathrm{~T}$ Signa scanner (GE Medical Systems, Milwauke, WI). All DTI images acquired consisted of 26 volumes (35 slices, image matrix size $256 \times 256$ voxels, field of view 
(FOV) $24 \mathrm{~cm}$, slice thickness $3 \mathrm{~mm}$, voxel size $0.9375 \times 0.9375 \times 3 \mathrm{~mm}^{3}$ ) representing 25 gradient field directions and one scan without a gradient field. Echo time (TE) and repetition time (TR) were $82.5 \mathrm{~ms}$ and $10,000 \mathrm{~ms}$, respectively. The $b$-value was $1000 \mathrm{~s} / \mathrm{mm}^{2}$.

\subsection{Image Processing}

Image reconstruction and processing was performed in AFNI (Cox, 1996) and FSL (Smith et al.,

2004; Woolrich et al., 2009). For an accurate determination of diffusion indices, all images were pre-processed to eliminate artifacts caused by subject motion and eddy currents. While head motion mostly causes rigid-body shifts and rotations, eddy currents can induce misalignments of the acquired images and miscalculations of DTI parameters (Horsfield, 1999). In order to correct for head motion, all images were aligned using the AFNI co-registration tool (Cox,

Jesmanowicz, 1999). Following alignment, the resulting DTI images were preprocessed in FSL to remove image distortions resulting from eddy currents. After data alignment the BET brain extraction tool (Smith, 2002) was applied to the $\mathrm{S}_{0}$ (with no diffusion weighting) volume to exclude non-brain voxels from further analysis. Then a 3x3 diffusion tensor $\mathbf{D}$ was fitted at each voxel using the DTIfit tool in FSL. The diffusion tensors at each voxel were diagonalized using multivariate fitting to obtain the eigenvectors and eigenvalues. Invariant scalar quantities,

namely fractional anisotropy (FA), mean diffusivity (MD), axial diffusivity $\left(\lambda_{\|}\right)$ and transverse diffusivity $\left(\lambda_{\mathrm{T}}\right)$ were calculated using DTIfit. In the next step all FA data were co-registered to $1 \times 1 \times 1 \mathrm{~mm}^{3}$ MNI152 space (Evans et al., 1993) by using the nonlinear registration Second, we perform tool FNIRT in FSL. The resulting FA volumes were merged into a single $4 \mathrm{D}$ file and averaged to create the mean of FA images. The mean FA image is then fed into the FA skeletonization program to create the mean FA skeleton, which contains the major white matter tracts common to all subjects. An example of the mean FA image with a threshold of 0.2 , and the mean FA skeleton is displayed in Fig. 1. Finally, the volumes were smoothed using convolution with an exponential kernel of fourth order and fullwidth half-maximum of $4 \mathrm{~mm}$. The volumes of the mean, transverse and axial diffusivity were transformed into the same space.

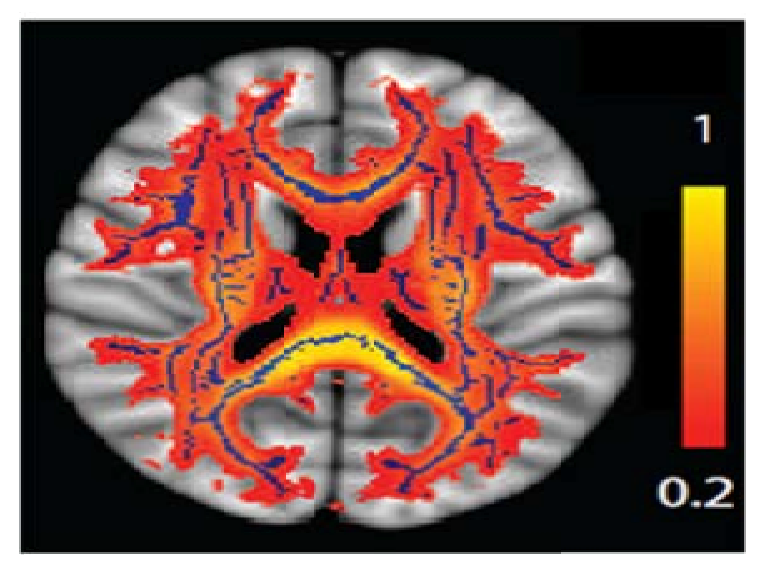

Figure 1. Example overlay of FA from 5 controls and 3 injured subjects after each volume has been nonlinearly aligned to the target in MNI152 space. The mean FA, shown in red-yellow, is thresholded at 0.2 ; the skeleton with a threshold of 0.3 is shown in blue.

\subsection{Statistical Analyses}

Statistical analysis of the data was performed in two ways: First, using TBSS (tract-based spatial statistics), which is part of the FSL package and based on the convoluted, skeletonized FA.

d student t-tests with multi-comparison correction to identify voxel in 
individual slices that were significantly different between the concussed group (CON) and the control groups (CGA and CGB). All tests were applied to all four diffusion indices.

\section{Results}

\subsection{Tract-based Spatial Statistics}

For all four diffusion indices group differences were calculated using tractbased spatial statistics (TBSS) (Nichols, Holmes, 2001; Smith et al., 2008) in FSL to identify voxels on the skeletonized data that are significantly different between the injured group (CON) and control group A (CGA) for the scan within 24 hours and the follow-up scans, as well as between control group A and control group B (CGB). Using the randomize routine in FSL, significant differences between the injured subjects and control group A at a level of $p<0.05$ were found from the first scan for FA, and the mean and transverse diffusivity. Axial diffusivity did not reach the significance level and neither did any quantity from the follow-up scans. As expected, the comparison between CGA and CGB did not return any significantly different voxels. The spatial regions, where significant differences were found, are shown in Fig. 2.

TBSS reveals a significant increase in fractional anisotropy and a decrease in transverse diffusivity in overlapping regions in the corticospinal tract in the right hemisphere in CON compared to CGA. Likewise, areas of significantly reduced mean diffusivity are found in the corticospinal tract in the left hemisphere.
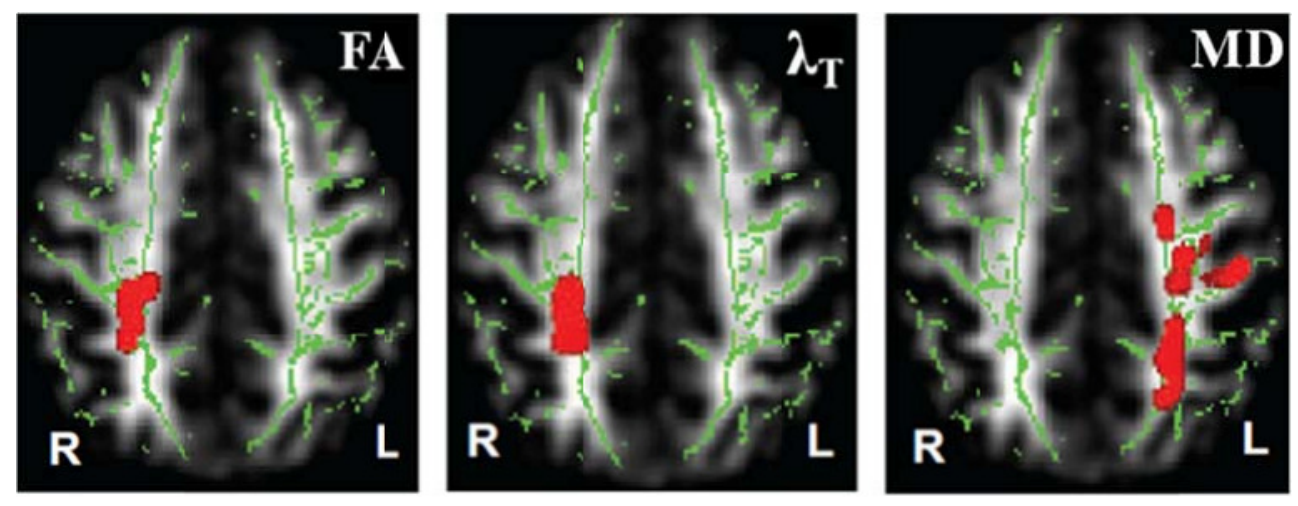

Figure 2. TBSS results for FA, $\lambda_{\mathrm{T}}$ and MD from 5 controls and 3 injured subjects with the mean FA skeleton (green) overlaid on top of the coregistered/convoluted FA. Regions of significant difference in concussed subjects compared to controls are shown in red.

\subsection{Voxel-wise T-tests}

As a second analysis, we applied voxel-wise t-tests, performed in MATLAB, to identify significantly different voxels between control group A and the injured group in all three scans, as well as between the two control groups CGA and CGB. In a first step, we counted the number of significantly different voxels in axial slices and plotted them as a function of the slice number. Here whole slices (after brain extraction) where used as regions of interest and 
correction for multiple comparison was performed by determining the minimum cluster size for a given slice at a significance level of $\mathrm{p}<0.05$ using the $3 \mathrm{dClustSim}$ routine, which is part of AFNI. The only significantly different regions that extend across more than one slice were located in the upper part of the brain, where differences were also found using tractbased statistics. We obtained the results shown in Fig. 3 for fractional anisotropy, and mean and transverse diffusivity. Red, green and blue curves show the number of different voxels between CGA and the first, second and

third scan of the injured group, respectively; no significantly large clusters were found for axial diffusivity and in comparison between CGA and CGB. FA, MD and $\lambda_{T}$ exhibit pronounced peaks for all three scans with maxima around slice \#115, the same brain region in the cortiospinal tract where differences were found using TBSS. As with TBSS, only the differences

for the first three quantities reached significance. Moreover, the number of different voxels for $\mathrm{MD}$ and $\lambda_{\mathrm{T}}$ clearly decreased between the first and the followup scans, pointing to a process of recovery.

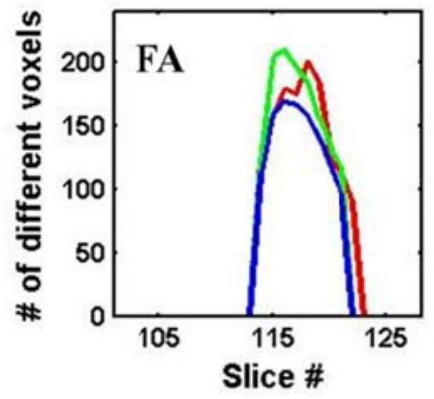

Figure 3. Number of voxels in axial slices that show a significant difference $(\mathrm{p}<0.05$, multi- comparison correction at the single slice level) in t-tests comparing $\mathrm{CON}$ to CGA in the first (red), second (green) and third (blue) scan in the upper region of the brain. Peaks with a maximum around slice \#115 are found for FA, MD and $\lambda_{\mathrm{T}}$ in the first scans and less pronounced in the follow-ups. No significant differences were found for axial diffusivity and between the two control groups.

The brain areas around axial slice \#115, where significant differences exist between
CON and CGA, are shown in Fig. 4. Differences in the first scan are indicated by the red regions, whereas the areas for the second and third (follow-up) scan are encircled by green and blue contour lines, respectively. The affected areas are similar to those identified by tract-based spatial statistics for $F A$ and $\lambda_{T}$ in the right hemisphere; for $\mathrm{MD}$ in the first scan differences were found in both hemispheres, for the follow-up scans only on the left. In particular for the mean and transverse diffusivity the extent of affected areas is reduced for the second and third scan, which is also visible, even though 

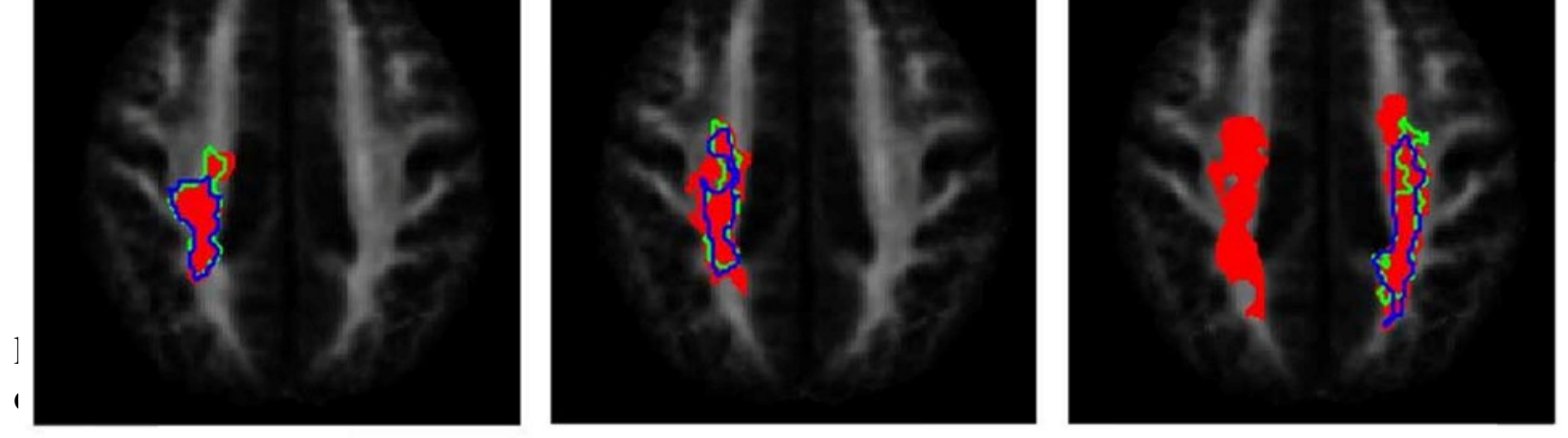

Figure 4. Spatial locations where significantly different clusters were found in a comparison of CON to CGA (multicomparison correction for a volume of 15 slices). Areas in red show differences in the first scan; regions encircled by green and blue contour lines correspond to scans two and three, respectively. Finally, we compared the normalized mean values of the four diffusion indices from voxels inside the affected regions for individual subjects. Fig. 5 shows the five control subjects in purple, and the injured group from the first, second and third scan in red, green and blue together with the group average (black dotted lines) and error bars indicating standard deviation. Single asterisks $(*)$ denote a significance level of $\mathrm{p}<0.05$ and double asterisks (**) stand for $\mathrm{p}<0.005$.

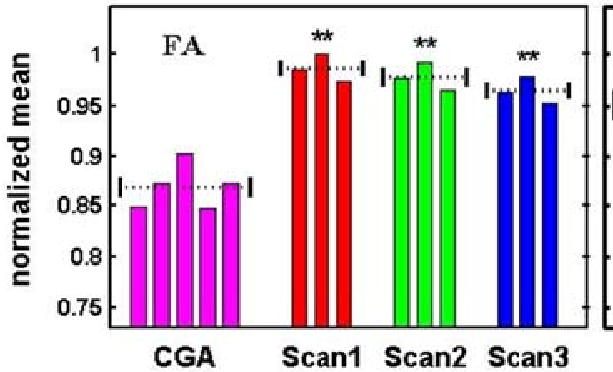

Figure 5. Normalized mean values for all diffusion indices and scans from voxels inside the affected areas for individual subjects from the control group (purple) and the injured group (red, green and blue) together with the group means (black dotted) and error bars showing standard deviation. The level of significance for differences between CGA and CON in the three scans is indicated by a single asterisk (*) for $\mathrm{p}<0.05$ and a double asterisk $(* *)$ increase

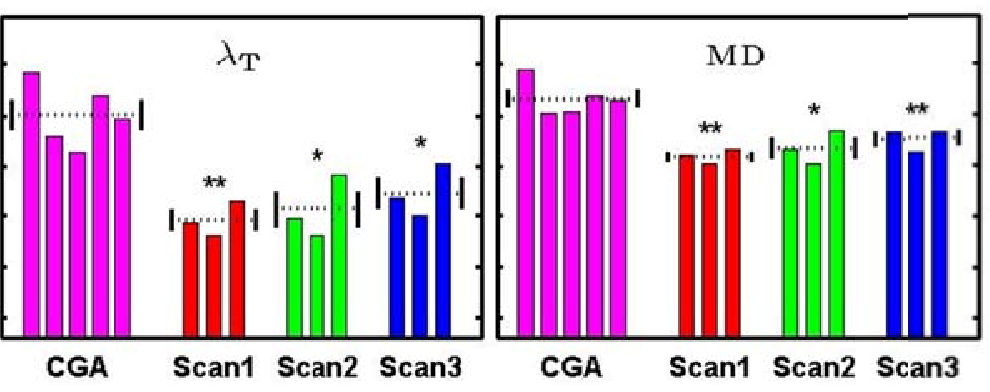

for $\mathrm{p}<0.005$. The histograms are normalized such that the largest value for all subjects and scans for each diffusion index is set to 1.

The plots are normalized such that for each diffusion index the maximum value from all subjects and scans is set to 1 . In agreement with the findings from TBSS, fractional anisotropy is in the affected regions after the injury, whereas mean and transverse diffusivity are 
decreased. Moreover, for all indices showing significant differences and all individual subjects from CON (as well as the group means) there is a shift toward the values from the CGA group, even though the affected areas for mean and axial diffusivity are smaller in scans two and three. This means that although the voxels inside the shrinking regions still show a significant difference on the group level, the actual values for the diffusion indices relax back toward normal control levels.

\section{Discussion}

Diffusion tensor imaging has now been used for roughly 10 years as a sensitive imaging tool for studying abnormalities in the brain in patients suffering from traumatic brain injuries. In a recent review, Hulkower et al. (2013) list and summarize the findings from 100 articles on DTI of mild to severe traumatic brain injuries. The variety of causes of the injury, its severity, different scan and analysis protocols and time past between the injury and the scan(s) leads to a broad range of brain locations where abnormalities are found, as well as whether the abnormality manifests itself as an increase or decrease in diffusive indices. In the majority of articles a decrease in FA and an increase in MD is reported, which is contrary to our findings. However, in several studies (Chu et al., 2010; Henry et al., 2011; Mayer et al, 2010) the results are similar to ours and it is argued that such findings of an increased FA and a decreased MD postinjury are most common for the acute phase of mild traumatic injuries in young patients and possibly due to cytotoxic cerebral edema.

Even though the number of injured subjects in our study is quite small, there is the advantage that the injuries occurred in very similar situations during football play or practice. Whereas in most studies MTBI patients from motor vehicle accidents, falls, assaults and other causes are typically pooled together, our three cases are relatively controlled as all subjects were wearing helmets and consequently had no localized or open head injuries. Moreover, the time past injury when the scans were performed was the same for all of them, which may explain the consistency in the affected locations and the recovery process. The strategy in this study was to identify affected brain regions by comparison of two groups (the three injured players and the five controls) and then look into these regions on an individual basis. As it turned out, all the diffusion indices above were different from the controls by several standard deviations for all individuals, which means that these specific regions were affected in all of them and therefore were significantly different on the group level. This does not mean that there are no other affected areas in individuals that were not significant as a group.

Two points from our results are of particular importance regarding the usefulness of DTI as a diagnostic tool for mild traumatic brain injuries and monitoring recovery: First, the mean FA $\left(\mathrm{MD}, \lambda_{\mathrm{T}}\right)$ values from the scan within 24 hours for the individual injured subjects were all substantially larger (smaller) than the largest (smallest) value from the control group in affected voxels. Second, there is a remarkable consistency and reproducibility 
for the individual subjects and scans for these diffusion indices: Subject \#2 (plotted in the middle) shows the largest and subject \#3 (right) shows the smallest deviation from the controls in all scans. Taken together this means that it may be possible to identify injured brain regions on an individual basis, a necessity if DTI is to qualify as a clinical diagnostic tool, by comparison to a sufficiently large set of controls.
In short, our results show that diffusion tensor imaging is a powerful technique for early detection of axonal injuries and may serve as an important tool for monitoring microstructural changes during recovery from MTBI.

\section{Acknowledgement}

Work supported by NINDS grant 48229 to JASK 


\section{References}

Adams, J.H., Doyle, D., Ford, I., Gennarelli, T.A., Graham, D.I., McClellan, D.R., 1989. Diffuse axonal injury in head injury: definitions, diagnosis and grading. Histopathology 15:49-59.

Alves, W., Macciocchi, S., Barth, J.T., 1993. Postconcussive symptoms after uncomplicated mild head injury. J Head Trauma Rehab 8, 48-59.

Bazarian, J.J., McClung, J., Shah, M.N., Cheng, Y.T., Flesher, W., Kraus, J., 2005. Mild traumatic brain injury in the United States, 1998-2000. Brain Injury 19, 8591.

Bazarian, J.J., Zhong, J., Blyth, B., Zhu, T., Kavcic, V., Peterson, D., 2007. Diffusion tensor imaging detects clinically important axonal damage after mild traumatic brain injury: A pilot study. J Neurotrauma 24, 14471459.

Chu, Z., Wilde, E.A., Hunter, J.V., McCauley, S.R., Bigler, E.D., Troyanskaya, M., Yallampalli, R., Chia, J.M., and Levin, H.S., 2010. Voxel-Based Analysis of Diffusion Tensor Imaging in Mild Traumatic Brain Injury in Adolescents. Am J Neuroradiol 31, 340346.

Cox, R.W., 1996. AFNI: Software for analysis and visualization of functional magnetic resonance Neuroimages. Comput Biomed Res 29, 162-173.
Cox, R.W., Jesmanowicz, A., 1999. Realtime 3D image registration for functional MRI. Magn

Reson Med 42, 1014-1018.

Evans, A., Collins, D., Mills, S., Brown, E., Kelly, R., Peters, T., 1993. 3D statistical neuroanatomical models from 305 MRI volumes. In: Nuclear Science Symposium and Medical Imaging Conference. 1993 IEEE Conference Record 1813-1817.

Gennarelli, T.A., 1986. Mechanisms and pathophysiology of cerebral concussion. J Head

Trauma Rehab 1, 23-29.

Henry, L.C., Tremblay, J., Tremblay, S., Lee, A., Brun, C., Lepore, N., Ellemberg, D., Lassonde, M., 2011. Acute and Chronic Changes in Diffusivity Measures after Sports Concussion. J Neurotrauma 28, 2049-2059.

Horsfield, M.A., 1999. Mapping eddy current induced field for the correction of diffusion weighted echo planar images. Magn Reson Imaging 17, 1335-1345.

Hulkower, M.B., Poliak, D.B., Rosenbaum, S.B., Zimmermann, M.E., Lipton, M.L., 2013. A Decade of DTI in Traumatic Brain Injury: 10 Years and 100 Articles Later. Am J Neuroradiol 34:2064-2074.

Jantzen, K.J., Anderson, B., Steinberg, F.L., Kelso, J.A.S., 2004. A prospective MR imaging study of mild traumatic 
brain injury in college football players. Am J Neuroradiol 25, 738-745

Mayer, A.R., Ling, J., Mannell, M.V., Gasparovic, C., Phillips, J.P., Doezema, D., Reichard, R., Yeo, R.A., 2010. A prospective diffusion tensor imaging study in mild traumatic brain injury. Neurology 74, 643-650.

Nichols, T.E., Holmes, A.P., 2001. Nonparametric permutation tests for functional neuroimaging: a primer with examples. Hum Brain Mapp 15, 1-25.

Pearce, J.M.S., 2008. Observations on concussion. Eur Neurol 59, 113-119.

Povlishock, J.T., Becker, D.P., Cheng, C.L., Vaughan, G.W., 1983. Axonal change in minor head injury. $J$ Neuropathol Exp Neurol 42, 225-242.

Ropper, A.H., Gorson, K.C., 2007. Concussion. N Engl J Med 356, 166-172.

Smith, D.H., Meaney, D.F., 2000. Axonal damage in traumatic brain injury. Neuroscientist 6, 483-495.

Smith, S.M., 2002. Fast robust automated brain extraction. Hum Brain Mapp 17,
143-155. Smith, S.M., Jenkinson, M.,

Woolrich, M.W., Beckmann, C.F., Behrens, T.E.J., Johansen-Berg, H., Bannister, P.R., De Luca, M., Drobnjak, I., Flitney, D.E., Niazy, R., Saunders, J., Vickers, J., Zhang, Y., De Stefano, N., Brady, J.M., Matthews, P.M., 2004. Advances in functional and structural MR image analysis and implementation as FSL. Neuroimage 23(S1), 208-219.

Smith, S.M., Jenkinson, M., JohansenBerg, H., Rueckert, D., Nichols, T.E., Mackay, C.E., Watkins, K.E., Ciccarelli, O., Cader, M.Z., Mathews, P.M., Behrens, T.E.J., 2008. Tract-based spatial statistics: voxelwise analysis of multi-subject diffusion data. Neuroimage 31, 1487-1505.

Woolrich, M.W., Jbabdi, S., Patenaude, B., Chappell, M., Makni, S., Behrens, T., Beckmann, C., Jenkinson, M., Smith, S.M., 2009. Bayesian analysis of neuroimaging data in FSL. Neuroimage

45(S1), 73-186.

Xiong, Y., Mahmood, A., Chopp, M., 2013. Animal models of traumatic brain injury. Nat Rev

Neurosci 14, 128-142. 